\title{
MODELLING AND SIMULATION OF THE EFFECT OF BLAST LOADING ON STRUCTURES USING AN ADAPTIVE BLENDING OF DISCRETE AND FINITE ELEMENT METHODS
}

\author{
CARLOS LABRA*, EUGENIO OÑATE*, FRANCISCO ZÁRATE*, JERZY \\ ROJEK $^{*, \dagger}$ \\ * Centre Internacional de Mètodes Numèrics en Enginyeria (CIMNE) \\ Universitat Politècnica de Catalunya (UPC) \\ Campus Nord UPC, 08034 Barcelona, Spain
}

e-mail: clabra@cimne.upc.edu, onate@cimne.upc.edu,zarate@cimne.upc.edu,www.cimne.com/
$\dagger$ Institute of Fundamental Technological Research Polish Academy of Sciences
Swietokrzyska 21, PL-00049. Warsaw, Poland e-mail: jrojek@ippt.gov.pl

Key words: Blasting, Discrete elements, Finite elements, Coupling

\begin{abstract}
We present a new computational model for predicting the effect of blast loading on structures. The model is based in the adaptive coupling of the finite element method (FEM) and the discrete element method (DEM) for the accurate reproduction of multifracturing and failure of structures under blast loading. In the paper we briefly describe the basis of the coupled DEM/FEM technology and demonstrate its efficiency in its application to the study of the effect of blast loading on a masonry wall, a masonry tunnel and a double curvature dam.
\end{abstract}

\section{INTRODUCTION}

The paper presents a procedure for modelling and simulation of the effect of blast loading on structures via the adaptive coupling of the discrete element method and the finite element methods. The theoretical formulation of the discrete element method using spherical or cylindrical particles is briefly reviewed. The finite element equations for structural dynamics are integrated using a standard explicit time integration scheme. The formulation of an adaptive multiscale DEM/FEM model employing the DEM and FEM in different subdomains of the same body is presented. An overlap zone in the DEM and FEM domains is introduced adaptively in order to provide a smooth transition from one discretization method to the other. Coupling between the DEM and FEM overlapping 
subdomains is provided by kinematic constraints imposed via a penalty function method. The efficiency of the new DEM/FEM method is demonstrated in its application to the study of the effect of blast loading on a masonry wall, a masonry tunnel and a double curvature concrete dam.

\section{DISCRETE ELEMENT METHOD FORMULATION}

The discrete element method (DEM) is widely recognized as a suitable tool to model geomaterials. This procedure can also be effectively used to model multifracture in "continuum" structures modelled as a collection of discrete elements. Formulation of spherical discrete elements following the main assumptions of Cundall $[1,2]$ has been developed by Oñate and Rojek [5] and Rojek and Oñate [8,9] and implemented in an explicit dynamic formulation. The DEM assumes that the solid material can be represented as a collection of rigid particles (spheres or balls in 3D and discs in 2D) interacting with each other in the normal and tangential directions at the contact points. Material deformation is assumed to be concentrated at the contact points. Appropriate contact laws allow us to obtain desired macroscopic material properties. The contact law used takes into account cohesive bonds between rigid particles. Cohesive bonds can be broken, thus allowing to simulate fracture of material and its propagation.

\subsection{Equations of motion}

The translational and rotational motion of rigid spherical (3D) or cylindrical (2D) elements (particles) is described by means of the standard equations of rigid body dynamics. For the $i$-th element we have

$$
m_{i} \ddot{\mathbf{u}}_{i}=\mathbf{F}_{i} \quad, \quad I_{i} \dot{\boldsymbol{\omega}}_{i}=\mathbf{T}_{i}
$$

where $\mathbf{u}$ is the displacement of the element centroid in a fixed (inertial) coordinate frame $\mathbf{X}, \boldsymbol{\omega}$ - the angular velocity, $m$ - the element mass, $I$ - the moment of inertia, $\mathbf{F}$ - the resultant force, and $\mathbf{T}$ - the resultant moment about the central axes. Vectors $\mathbf{F}$ and $\mathbf{T}$ include all external forces and moments applied to the $i$-th element, contact forces due to interactions with neighboring element and other obstacles, as well as forces resulting from damping in the system.

The equations of motion (1) are integrated in time using an explicit central difference scheme. For the $i$ th element this gives:

$$
\ddot{\mathbf{u}}_{i}^{n}=\frac{\mathbf{F}_{i}^{n}}{m_{i}} \quad, \quad \dot{\mathbf{u}}_{i}^{n+1 / 2}=\dot{\mathbf{u}}_{i}^{n-1 / 2}+\ddot{\mathbf{u}}_{i}^{n} \Delta t \quad, \quad \mathbf{u}_{i}^{n+1}=\mathbf{u}_{i}^{n}+\dot{\mathbf{u}}_{i}^{n+1 / 2} \Delta t .
$$

The first two steps in the integration scheme for the rotational motion are identical to those given by Equation (2):

$$
\dot{\boldsymbol{\omega}}_{i}^{n}=\frac{\mathbf{T}_{i}^{n}}{I_{i}} \quad, \quad \boldsymbol{\omega}_{i}^{n+1 / 2}=\boldsymbol{\omega}_{i}^{n-1 / 2}+\dot{\boldsymbol{\omega}}_{i}^{n} \Delta t
$$




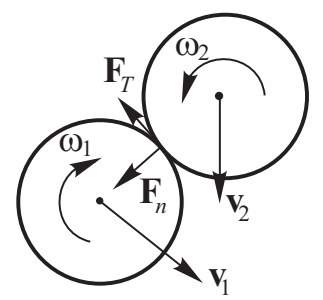

(a)

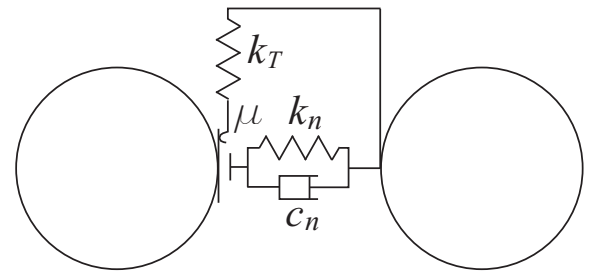

(b)

Figure 1: (a) Decomposition of contact force in normal and tangential components. (b) Model of the contact interface.

The vector of incremental rotation $\Delta \boldsymbol{\theta}=\left\{\Delta \theta_{x} \Delta \theta_{y} \Delta \theta_{z}\right\}^{\mathrm{T}}$ is calculated as $\Delta \boldsymbol{\theta}_{i}=$ $\omega_{i}^{n+1 / 2} \Delta t$.

Knowledge of the incremental rotation suffices to update the tangential contact forces. It is also possible to track the rotational position of particles, if necessary. The rotation matrices between the moving frames embedded in the particles and the fixed global frame are updated incrementally using a multiplicative scheme [5, 8, 9].

\subsection{Contact search algorithm}

Changing contact pairs of elements during the analysis process must be automatically detected. The simple approach to identify interaction pairs by checking every sphere against every other sphere would be very inefficient, as the computational time is proportional to $n^{2}$, where $n$ is the number of elements. In our formulation the search is based on quad-tree and oct-tree structures. In this case the computation time of the contact search is proportional to $n \ln n$, which allows to solve large frictional contact systems.

\subsection{Evaluation of contact forces}

Once contact between a pair of elements is detected, the forces occurring at the contact point are calculated. The interaction between the two interacting bodies can be represented by the contact forces $\mathbf{F}_{1}$ and $\mathbf{F}_{2}$, which by the Newton's third law satisfy the following relation:

$$
\mathbf{F}_{1}=-\mathbf{F}_{2}
$$

We take $\mathbf{F}=\mathbf{F}_{1}$ and decompose $\mathbf{F}$ into the normal and tangential components, $\mathbf{F}_{n}$ and $\mathbf{F}_{T}$, respectively as $\mathbf{F}=\mathbf{F}_{n}+\mathbf{F}_{T}=F_{n} \mathbf{n}+\mathbf{F}_{T}$, where $\mathbf{n}$ is the unit vector normal to the particle surface at the contact point (Fig. 1a).

The value of the contact forces $F_{n}$ and $\mathbf{F}_{T}$ is obtained using a constitutive model formulated for the contact between two rigid elements. The contact interface in our formulation is characterized by the normal and tangential stiffness $k_{n}$ and $k_{T}$, respectively, the Coulomb friction coefficient $\mu$, and the contact damping coefficient $c_{n}$ (Fig. 1b). For details see $[5,8,9]$. 


\section{FINITE ELEMENT FORMULATION}

In the present work the so-called explicit dynamic formulation of the finite element equations of structural dynamics is used. The discretized equations of motion in the current configuration have the following form:

$$
\mathbf{M}_{\mathrm{F}} \ddot{\mathbf{u}}_{\mathrm{F}}=\mathbf{F}_{\mathrm{F}}^{\mathrm{ext}}-\mathbf{F}_{\mathrm{F}}^{\mathrm{int}}
$$

where $\mathbf{M}_{\mathrm{F}}$ is the mass matrix, $\mathbf{r}_{\mathrm{F}}$ is the vector of nodal displacements in the finite element mesh, $\mathbf{F}_{\mathrm{F}}^{\text {ext }}$ and $\mathbf{F}_{\mathrm{F}}^{\text {int }}$ are the vectors of external loads and internal forces, respectively. The global matrices and vectors, $\mathbf{M}_{\mathrm{F}}, \mathbf{F}_{\mathrm{F}}^{\mathrm{ext}}$ and $\mathbf{F}_{\mathrm{F}}^{\mathrm{int}}$, are assembled from the respective elemental matrices and vectors, $\mathbf{m}_{e}, \mathbf{f}_{e}^{\text {ext }}$ and $\mathbf{f}_{e}^{\text {int }}$, defined as follows:

$$
\mathbf{m}_{e}=\int_{\Omega_{e}} \rho \mathbf{N}^{\mathrm{T}} \mathbf{N} \mathrm{d} \Omega_{e} \quad, \quad \mathbf{f}_{e}^{\mathrm{int}}=\int_{\Omega_{e}} \mathbf{B}^{\mathrm{T}} \boldsymbol{\sigma} \mathrm{d} \Omega_{e} \quad, \quad \mathbf{f}_{e}^{\text {ext }}=\int_{\Omega_{e}} \mathbf{N}^{\mathrm{T}} \rho \mathbf{b} \mathrm{d} \Omega_{e}+\int_{\Gamma_{e}} \mathbf{N}^{\mathrm{T}} \mathbf{t} \mathrm{d} \Gamma_{e}
$$

where $\rho$ is the mass density, $\boldsymbol{\sigma}$ is the Cauchy stress tensor, $\mathbf{b}$ are the body forces, $\mathbf{t}$ is the surface traction, $\mathbf{N}$ is the matrix of interpolation (shape) functions and $\mathbf{B}$ is the linear strain-displacement operator matrix [8].

Similarly to the DEM algorithm (Equation (2)), the central difference scheme is used for time integration of Equation (5):

$$
\ddot{\mathbf{u}}_{\mathrm{F}}^{n}=\mathbf{M}_{\mathrm{F}}^{-1}\left(\mathbf{F}_{\mathrm{F}}^{\mathrm{ext}}-\mathbf{F}_{\mathrm{F}}^{\mathrm{int}}\right)^{n} \quad, \quad \dot{\mathbf{u}}_{\mathrm{F}}^{n+1 / 2}=\dot{\mathbf{u}}_{\mathrm{F}}^{n-1 / 2}+\ddot{\mathbf{u}}_{\mathrm{F}}^{n} \Delta t \quad, \quad \mathbf{u}_{\mathrm{F}}^{n+1}=\mathbf{u}_{\mathrm{F}}^{n}+\dot{\mathbf{u}}_{\mathrm{F}}^{n+1 / 2} \Delta t .
$$

Use of a diagonalized mass matrix yields a decoupled set of equations, and eliminates the necessity of matrix inversion in Equation (7.1). This leads to a very efficient solution for each time step. For details see [5, 8, 9].

\section{COMBINED DEM/FEM MODEL}

The coupling of DEM and FEM techniques leads to a powerful scheme for analysis of multifracture problems in solids. Successful attempts to develop a coupled DEM/FEM algorithm have been reported in [5, 4]. The adaptive DEM/FEM multiscale model used in this work is obtained by combining the discrete element and finite element methods in different subdomains of the same body. The coupling algorithm used here follows the concept presented in [10] for molecular dynamics coupling with a continuous model. The DEM and FEM subdomains can overlap each other. In this way a transitory zone between the microscopic-scale zone (discrete elements)and the macroscopic-scale zone (finite elements) is introduced. In the overlapping zone contributions of each of the two methods to the overall stiffness vary gradually. This allows us to avoid or minimize unrealistic wave reflections at the interface between the DEM and FEM domains.

The total domain $\Omega$ is split into two subdomains: $\Omega_{F}$, discretized with finite elements and $\Omega_{D}$, modelled with discrete elements. Domains $\Omega_{F}$ and $\Omega_{D}$ can overlap with each 


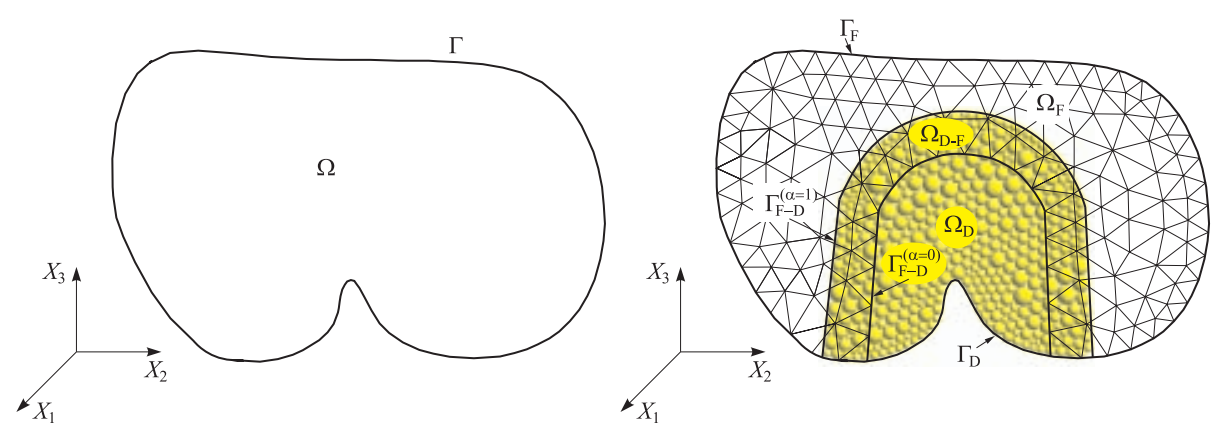

Figure 2: Schematic representation of DEM, FEM and DEM/FEM overlapping domains.

other over a region $\Omega_{D F}$ (Fig. 2). The virtual work (VW) in the total domain $\Omega\left(\delta W_{\Omega}\right)$ is written as a linear combination of the virtual work in the subdomains $\Omega_{F}$ and $\Omega_{D}$, i.e.

$$
\delta W_{\Omega}=\alpha \delta W_{F}=(1-\alpha) \delta W_{D}
$$

where $\alpha$ is a parameter that takes a zero value on $\Omega_{D}$, a unit value on $\Omega_{F}$ and it varies linearly between 0 and 1 on the overlapping region $\Omega_{D-F}$.

Subdomains $\Omega_{F}$ and $\Omega_{D}$ are coupled in the overlapping region $\Omega_{D-F}$. Coupling is introduced via kinematic constraints relating the displacements $(\mathbf{u})$, velocities $(\dot{\mathbf{u}})$ and accelerations $(\ddot{\mathbf{u}})$ of the nodes of the finite element mesh and the discrete elements belonging to $\Omega_{D-F}$. The kinematic constraint can be generically written as

$$
\delta \mathbf{u}_{D_{i}}-\mathbf{N}_{\mathrm{F}} \delta \mathbf{u}_{\mathrm{F}}=0 \quad, \quad \dot{\mathbf{u}}_{D_{i}}-\mathbf{N}_{\mathrm{F}} \dot{\mathbf{u}}_{\mathrm{F}}=0 \quad, \quad \ddot{\mathbf{u}}_{D_{i}}-\mathbf{N}_{\mathrm{F}} \ddot{\mathbf{u}}_{\mathrm{F}}=0
$$

where $(\cdot)_{D_{i}}$ and $(\cdot)_{F}$ respectively denote values at the discrete element $i$ and the finite element mesh nodes and $\mathbf{N}_{F}$ are the standard FEM shape functions. The constraints (9) are applied on the overlapping region $\Omega_{D-F}$ only.

The constraints (9) are introduced in the VW equation via a penalty function method. For details see [9].

\section{ADAPTIVE DEM-FEM SCHEME}

An adaptive DEM/FEM solution procedure has been developed based on the progressive introduction of discrete elements in zones of the finite element mesh where cracking and multifracture occurs. This optimizes the use of discrete elements to zones where they can be more efective which considerably simplifies the contact search process.

In essence, the adaptive DEM/FEM procedure operates as follows:

1. Start with a discretization of the analysis domain using a finite element mesh only (i.e. ${ }^{\circ} \Omega \equiv{ }^{\circ} \Omega_{F}$ ).

2. At each time instant $(t)$ check the stress and strain levels at each element. For linear triangles and tetrahedra this simple implies computing the strains and stresses at the element centroid. 
3. Evaluate the threshold of failure (fracture) of each element. This can be done via procedures based on the point-wise value of the stresses (or the strains), or using an adequate energy norm. In our work a simple Tresca failure model has been used to define the onset of fracture at the element mid-point.

4. Introduce a collection of discrete elements within the finite elements that have exceeded the failure threshold. In our work this occurs when the stresses at the element mid-point reach $90 \%$ of the Tresca failure stress. At this moment, the continuum region previously occupied by finite elements is now modelled with a collection of discrete elements.

The introduction of discrete elements will create an overlapping between the new discrete elements and the finite elements remaining in the mesh. Overlap DEM/FEM regions are treated as explained in the previous section.

5. Solve for the displacements, velocities and accelerations of the regions occupied with finite elements $\left(\Omega_{F}\right)$ and discrete elements $\left(\Omega_{D}\right)$ at $t+\Delta t$ using the explicit schemes (2) and (7) with the constraints (9).

6. The introduction of additional discrete element regions on the finite element mesh evolves in time in an adaptive manner accordingly to the evolution of the stress and strain fields in the analysis domain.

For blast problems the transition of the finite element mesh to the discrete element region occurs quite rapidly, as the fracture zone progresses almost at the blast speed on the whole analysis domain. However, the adaptive DEM/FEM procedure is still effective in these cases as the time increment for the explicit solution is very small and the delay in introducing discrete elements leads to considerable savings in computing time.

\section{EXAMPLES}

\subsection{Failure of a vertical wall due to blast loading}

This relatively simple $2 \mathrm{D}$ example shows the failure of a vertical masonry wall induced by a blast loading due to an explosive placed within a concrete box modelled with discrete elements (Fig. 3a). The effect of the explosive is simulated by an inpulse pressure load, with a peak pressure of $600 \mathrm{MPa}$ acting the center of the box. The explosion induces the multifracture of the box in many fragments that impact the adjacent wall inducing its instant failure (Fig. 4).

The cylinder wall has been initially discretized with a mesh of finite elements. The discrete elements have been progressively introduced in the wall using the adaptive DEM/FEM coupling algorithm. The evolution of the discrete element region in the wall is shown for three time instants in Figure 5.

Figure 6 shows a similar 2D problem for a masonry tunnel under the same type of blast loading. 


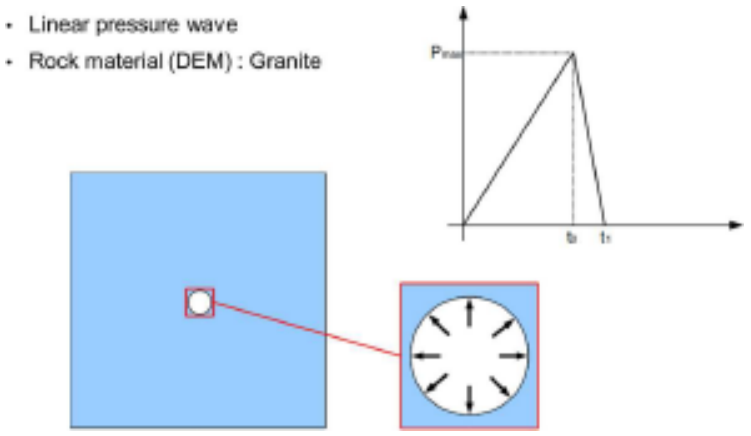

(a)

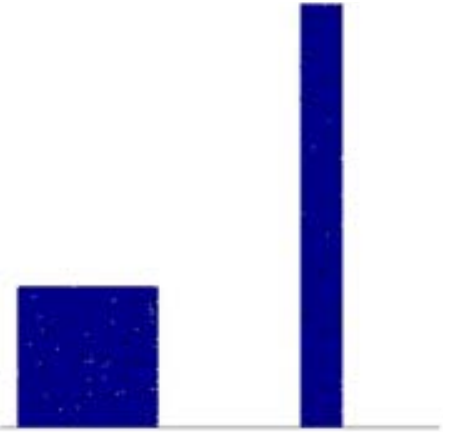

(b)

Figure 3: Masonry wall under blast loading induced by an explosive within a concrete box. (a) Concrete box and explosive condition, (b) Geometry of box and wall.
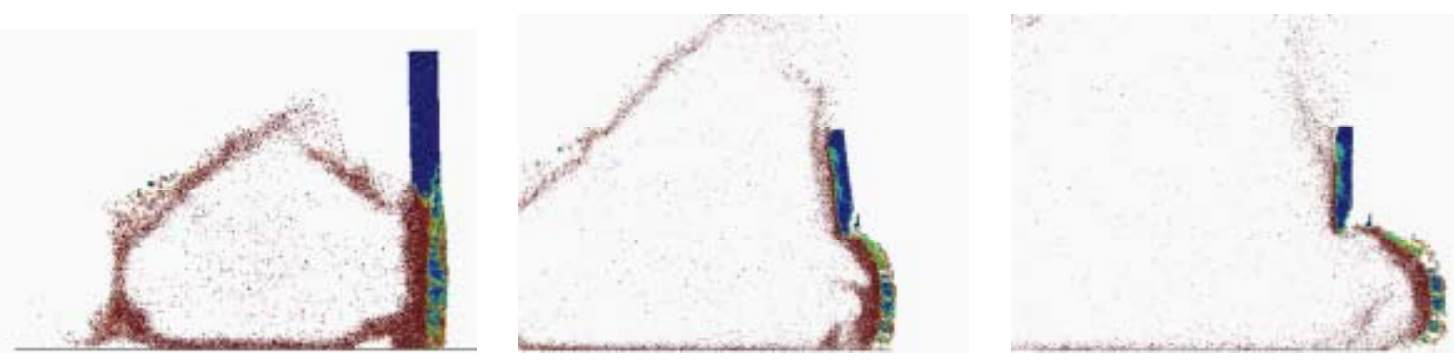

Figure 4: Masonry wall under blast load. Deformation of the wall at three time instants.
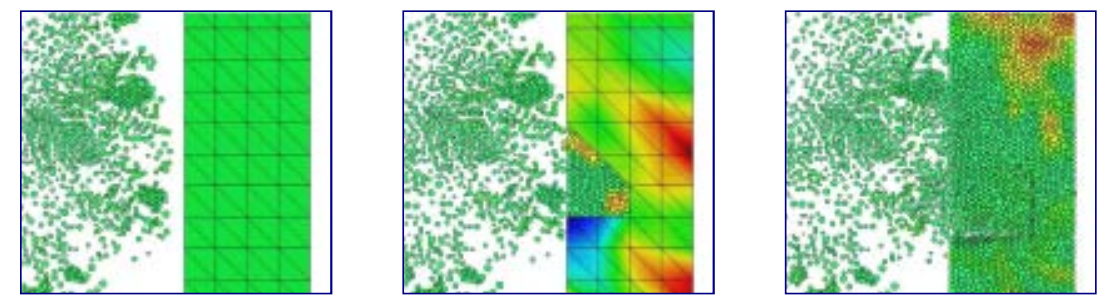

Figure 5: Detail of adaptive DEM/FEM procedure on the masonry wall at three time instants.

\subsection{Analysis of the fracture of a double curvature dam due to blast loading}

The final example is the study of the fracture induced by a blast load on a double curvature concrete dam. The load is induced by an explosive placed at the top of the central section, reproducing the effect of the explosion of a vehicle circulating over the dam top. The region adjacent to the explosion has been modelled with discrete (spherical) elements while the rest of the dam has been modelled with standard 4-noded tetrahedral elements.

Figure 7 shows the evolution of the fracture at the dam top due to the explosion and the final fractured zone. 

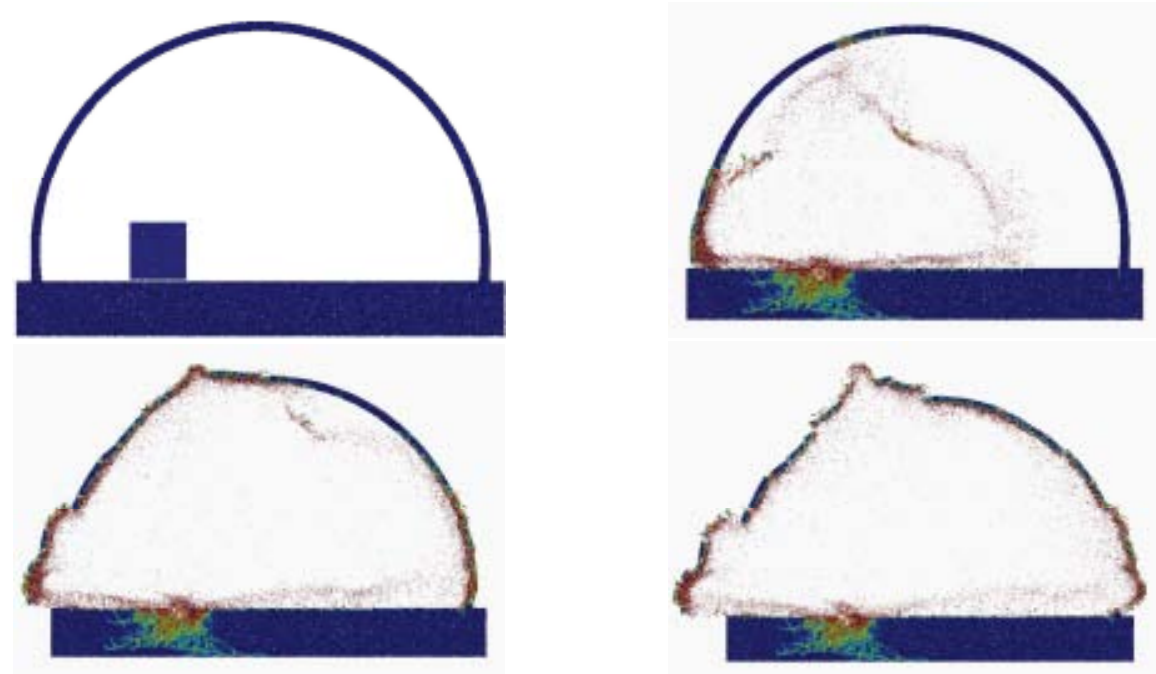

Figure 6: Masonry cylinder under internal blast load induced by an explosive within a concrete box. Deformation of structure at three time instants.
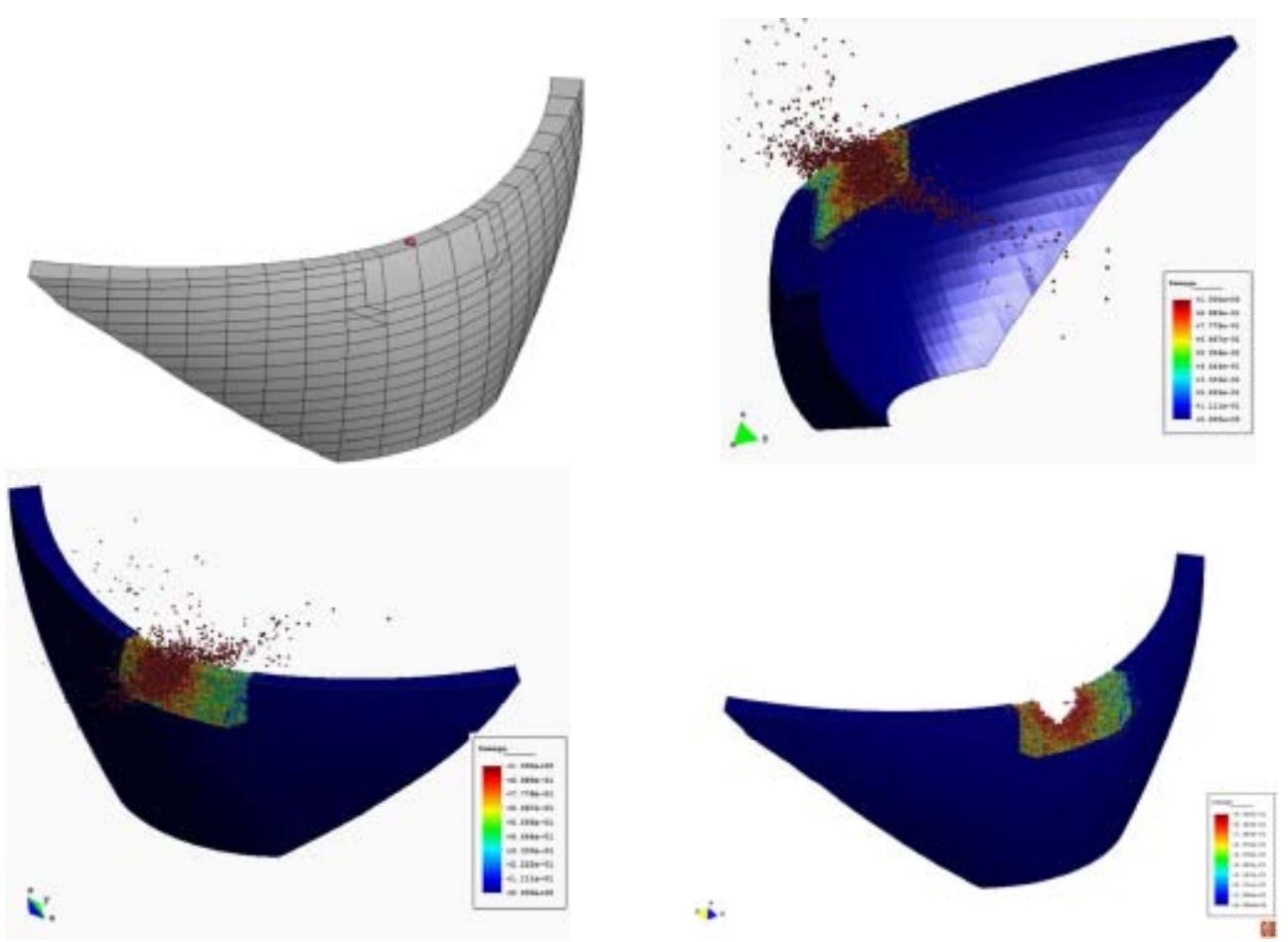

Figure 7: Effect of a blast load an a double curvature dam. The explosive load has been modelled as a peak pressure load of $600 \mathrm{MPa}$ acting at the center of the dam top.

\section{CONCLUDING REMARKS}

The adaptive DEM/FEM procedure presented in this work is an effective technique for the modelling and simulation of the progressive multi-fracture and failure of structures 
due to blast loading. The adaptive DEM/FEM scheme allows the optimal use of DEM and FEM in different parts of the structure as the failure region evolves.

\section{REFERENCES}

[1] Cundall, P.A. and Strack, O.D.L. 1979. A discrete numerical method for granular assemblies. Geotechnique 29:47-65.

[2] Cundall, P.A. 1988. Formulation of a Three Dimensional Distinct Element Model Part I. A Scheme to Detect and Represent Contacts in a System of Many Polyhedral Blocks. Int. J. Rock Mech., Min. Sci. and Geomech. Abstr. 25(3):107-116.

[3] Munjiza, A., Owen, D.R.J. and Bicanic, N. 1995. A combined finite-discrete element method in transient dynamics of fracturing solids. Engineeering Computations $12(2): 145-174$.

[4] Munjiza, A. 2004. The Combined Finite-Discrete Element Method. Wiley.

[5] Oñate, E. and Rojek, J. 2004. Combination of discrete element and finite element methods for dynamic analysis of geomechanics problems. Comput. Meth. Appl. Mech. Eng. 193:3087-3128.

[6] Oñate, E., Idelsohn, S.R., Celigueta, M.A., Rossi, R., Marti, J., Carbonell, J.M., Ryzhakov, P. and Suárez, B. 2011. Advances in the particle finite element method (PFEM) for solving coupled problems in engineering. E. Oñate and R. Owen (eds.), Particle-Based Methods. Published in Computational Methods in Applied Sciences, Vol. 25, pp 1-49, Springer.

[7] Oñate, E., Celigueta, M.A., Idelsohn, S.R., Salazar, F. and Suárez B. 2011. Possibilities of the Particle Finite Element Method for fluid-soil-structure interaction problems. Computational Mechanics. Accepted for publication.

[8] Rojek, J. and and Oñate, E. 2004. Unified DEM/FEM approach to geomechanics problems. In Proceedings of Computational Mechanics WCCM VI in conjunction with APCOM04, Beijing, China, Sept. 5-10.

[9] Rojek, J. and and Oñate, E. 2007. Multiscale analysis using a coupled discrete/finite element model. Interaction and Multiscale Mechanics 1(1):1-31.

[10] Xiao, S.P. and Belytschko, T. 2004. A bridging domain method for coupling continua with molecular dynamics. Comput. Meth. Appl. Mech. Eng. 193:1645-1669. 